\title{
Mobile Device Based Water Monitoring with Single Reference Image
}

\author{
Volkan Kılıç and Ali Y. Mutlu
}

\begin{abstract}
In this study, we report a low-cost and portable smartphone spectrometer system to monitor water quality based on the use of the built-in camera for capturing a single-use reference image. The images are spectrum view of the assays after flash light of the camera passes through the assays. The system processes the reference images in training step and creates a reference model for the calculation of unknown concentration assays in real experimental conditions. An experimental investigation for detection of methylene blue (MB) in water, relying on color change of the spectrum view due to concentration level, shows that the proposed system is able to calculate the concentration level accurately with a significantly reduced cost.
\end{abstract}

Index Terms - image processing, smartphone spectrometer, color matching.

\section{INTRODUCTION}

Colorimetry plays an important role in physical and analytical chemistry that aims to quantify the concentration of colored solutions [1]. Colorimeters quantify concentration level using absorbance measurements employing spectrophotometry and Beer-Lambert law [2] which found application areas in both laboratory and field use depending on the complexity of the experiment. With the help of 3D printers, smartphone spectrometer systems found wide range of colorimetric measurements application for chemical and biological analytes [3]. Some of them are water quality sensing for environment $[4,5]$, leaf color analysis for agriculture [6], $\mathrm{pH}[7,8]$ and glucose [9] sensing, and peanut allergen detection [10]. Color data is mostly used in paper based sensors application where parameters from various color spaces, i.e. RGB, HSV, and L*a*b* are used to quantify color change [11-14]. In this study, we propose a new methodology for simple smartphone based colorimetric detection of water quality running color-matching algorithms on JPEG images under well-defined experimental conditions. The proposed system is able to calibrate an entire set of concentrations using a single image which is further used to predict concentration of an unknown solution with great accuracy. The motivation here is to propose a portable, low-cost and user-friendly platform with a simple experimental methodology which has a quick response in detection. Moreover, it can be applied to any lab bench with no access to sophisticated equipment, and has the advantage to make the colorimetry test accessible for both regular and citizen scientists.

Volkan Kılıç and Ali Y. Mutlu both are with the Department of Electrical and Electronics Engineering, Izmir Katip Celebi University, Izmir, Turkey
The rest of this paper is organized as follows: the following section introduces experimental setup and methods that we use for colorimetric test. Section 3 presents experimental results and discussions. Closing remarks are given in Section 4.

\section{EXPERIMENTAL SETUP AND METHODS}

In this section, the experimental setup and methods are introduced. We start with a description of our custom-designed smartphone spectrometer before presenting the methods used for analyzing the results.

\section{A. Portable Smartphone Spectrometer Design}

The proposed portable smartphone spectrometer design is shown in Fig. 1a. The cradle was manufactured to integrate with LG G4 and the design was based on using rear camera of the smartphone to capture the spectrum view of the assays with $1 / 2.6$ " sensor size, 5312 x 2988 resolution and $1.12 \mu \mathrm{m}$ pixel size. In manufacturing process, a 3D printer (Zortrax M200) used the Acrylonitrile Butadiene Styrene polymer to fabricate a hot-plug apparatus toting a diffraction grating, and a smartphone case holder keeping the whole part compact with the cuvette holder with around use of $150 \mathrm{~g}$ polymer.

Two pieces of plastic fiber cables are used in the design. The first $1.5 \mathrm{~mm}$ diameter fiber carries the light from the smartphone flash to the cuvette while the second fiber cable with a diameter of $0.25 \mathrm{~mm}$ transmits the light from cuvette to the camera which passed through the assay. In order to simplify the spectrometer system design, no collector lens or mirrored components are placed in the light path. All components are mechanically aligned with the flash and camera. Besides cost, the most important factor in choosing plastic optical fibers instead of glass-based fiber optics is the ability to use plastic optical fibers without special tools for stripping and cutting.

\section{B. Image Pre-processing}

In experiments, LG G4 smartphone handset was kept in manual mode and the white balance, ISO, shutter speed and focus settings were remained constant throughout the experiments. The images captured by smartphone camera are in JPEG format with dimensions 5312 x 2988. Despite the size of the image, the region of interest, which is the data of the spectrum, occupies less than $1 \%$ of the entire image. Therefore, the input with only the region of interest will contribute to both the processing speed and the accuracy of the results. In the first step, the images were cropped to cover the spectrum data which leads to reduced image size of $1120 \times 99$. 


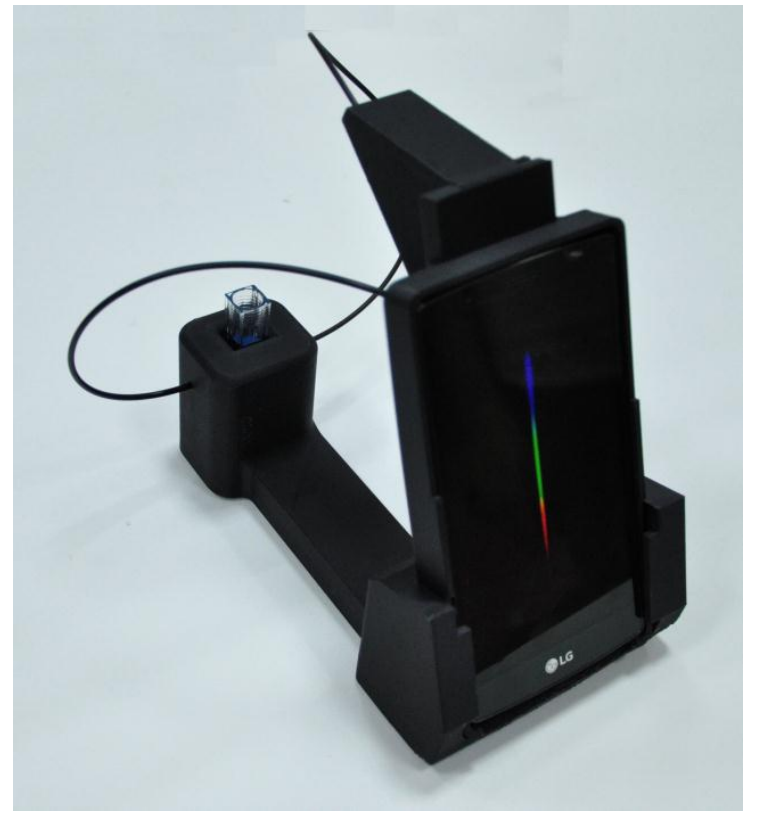

(a)

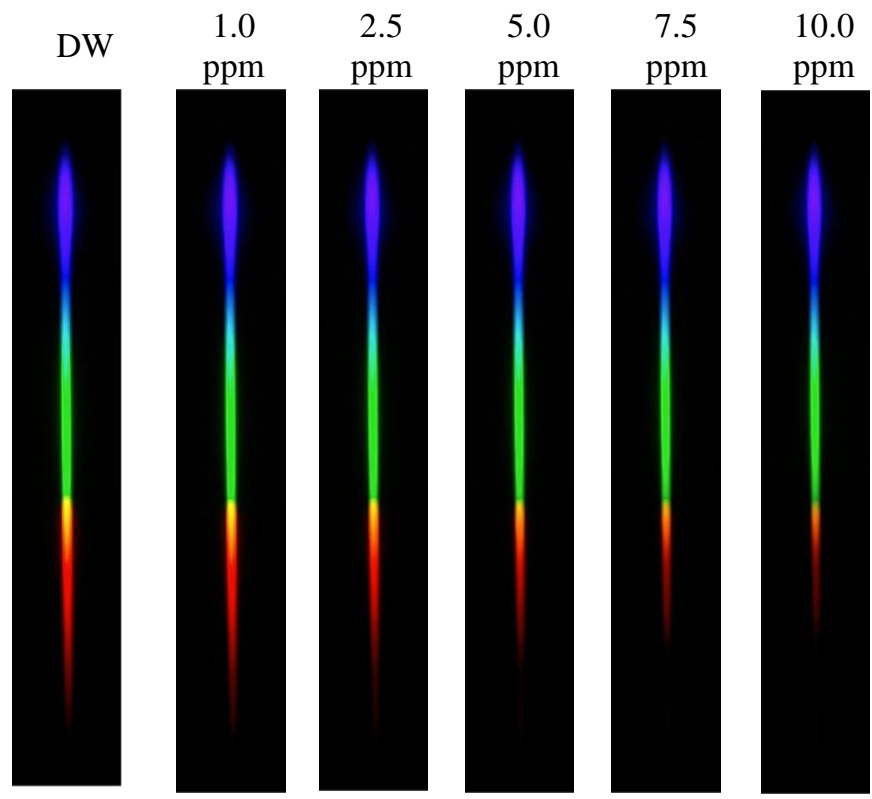

(b)

Fig. 1: Proposed smartphone spectrometer design is given in (a) and the spectrums of MB assays from 0 ppm (Distilled Water) to 10 ppm are given in (b).

This ratio was kept the same for all experiments and the cropped images are shown in Fig. 1b. Note that it is necessary to centralize the spectral data before cropping since the color matching algorithms make comparisons pixel to pixel. Therefore, we propose to choose the column with highest brightness for centralizing. As noticed from spectrum images from different concentrations, red region varies remarkably along with the density which enables the proposed approach to extract distinguished features from that region. For that reason, the cropped images were centralized by selecting the column with the highest brightness of red color density.

\section{DeltaE Method}

The first method used in colorimetric test is deltaE $\left(\Delta \mathrm{E}^{*}\right)$ method which is based on measuring color differences between two images using $\Delta \mathrm{E}^{*}$ distance metric obtained from the CIE $1976 \mathrm{~L}^{*} \mathrm{a} * \mathrm{~b} *$ color-difference formula [15]:

$$
\Delta E^{*}=\sqrt{\left(\Delta L^{*}\right)^{2}+\left(\Delta a^{*}\right)^{2}+\left(\Delta b^{*}\right)^{2}}
$$

Here, $\mathrm{L}^{*}, \mathrm{a}^{*}$ and $\mathrm{b}^{*}$ are dimensions of the CIE $\mathrm{L}^{*} \mathrm{a} * \mathrm{~b}^{*}$ color space where $\mathrm{L}^{*}$ axis defines lightness in the range of black (0) to white (100), and the $\mathrm{a}^{*}$ axis changes over red $\left(+\mathrm{a}^{*}\right)$ to green $\left(-\mathrm{a}^{*}\right)$, and the $\mathrm{b}^{*}$ axis defines yellow $\left(+\mathrm{b}^{*}\right)$ to blue $\left(-\mathrm{b}^{*}\right) . \Delta \mathrm{E}^{*}$ calculates a lower score for the similar images as the distance between similar images is smaller than the dissimilar images. In this study, all the images are converted to $\mathrm{L}^{*} \mathrm{a} * \mathrm{~b} *$ color space as it is more robust to illumination variations [16].

\section{Color-correlation Method}

The second method is color-correlation (CC) method [17] which calculates correlation coefficients between test image and trained images. The coefficient value varies between 0 and
1 where 1 represents the highest similarity, and the lowest similarity is represented by 0 . The correlation coefficient of reference image $I_{r e f}$ and test image $I_{\text {test }}$ is calculated as below:

$$
\rho\left(I_{\text {ref }}, I_{\text {test }}\right)=\frac{\operatorname{cov}\left(I_{\text {ref }}, I_{\text {test }}\right)}{\sigma_{\text {ref }} \sigma_{\text {test }}}
$$

where $\sigma_{\text {ref }}$ and $\sigma_{\text {test }}$ are the standard deviation of the reference and test images, and $\operatorname{cov}\left(I_{\text {ref }}, I_{\text {test }}\right)$ is the covariance of two images.

\section{RESULTS AND DISCUSSION}

For quantitative evaluation of smartphone based spectrometer system, methylene blue (MB) assays were prepared as 1.0, 2.5, 5.0, 7.5 and $10.0 \mathrm{ppm}$. Additionally, distilled water (DW) which corresponds to $0 \mathrm{ppm}$ was added. Then five consecutive images were taken from these six assays. For the training step, one reference image was selected out of five captured images in order to be used as a reference image. These reference images were saved with their concentration values. In the test step, individual image of each concentration is taken, and is sent to $\mathrm{CC}$ and $\Delta \mathrm{E}^{*}$ methods to detect the corresponding concentration value. The $\mathrm{CC}$ and $\Delta \mathrm{E}^{*}$ scores were calculated for each pixel of the test and reference images. Regularly used machine learning tools, i.e., confusion matrix, detection accuracy, precision, recall and f1-score, have been employed for measuring the performance of two algorithms. 
Fig. 2 shows the performance the $\Delta \mathrm{E}^{*}$ and $\mathrm{CC}$ methods in detecting MB concentration levels with confusion matrices where each row of the matrix represents the instances in an actual (true) class while each column represents the instances in a predicted class. The confusion matrices were created using 6 different concentration levels of MB. According to the deltaE Confusion Matrix

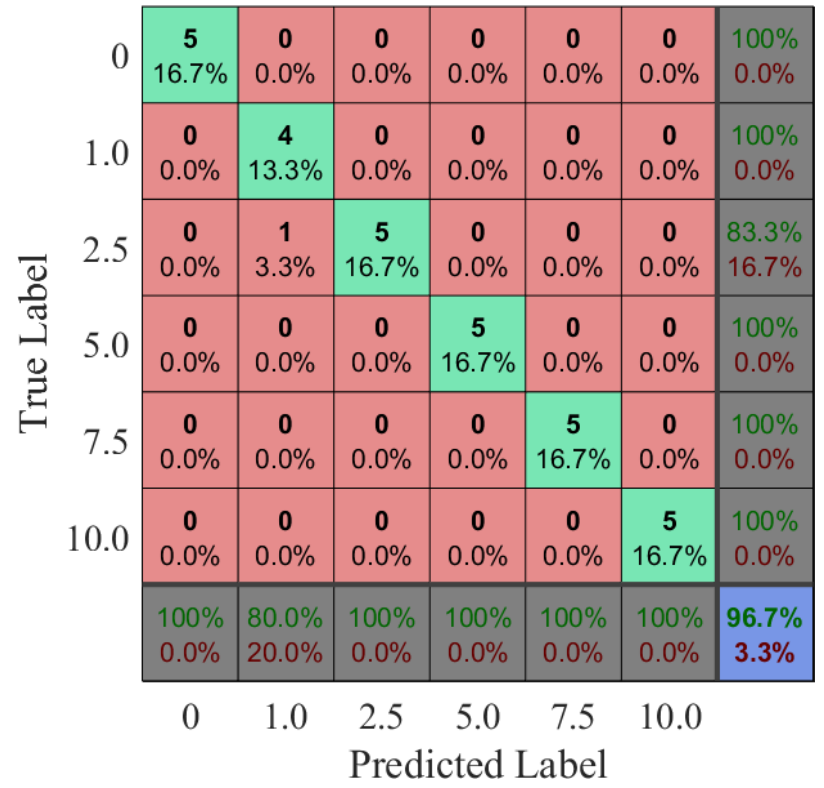

confusion matrices, $\Delta \mathrm{E}^{*}$ method outperforms the $\mathrm{CC}$ method in identifying the concentration levels of $\mathrm{MB}$, where there is only one misclassified concentration in $\Delta \mathrm{E}^{*}$ while it is two in the $\mathrm{CC}$ method. Furthermore, misclassification only happens between neighboring concentration values.

\begin{tabular}{|c|c|c|c|c|c|c|c|}
\hline 0 & $\begin{array}{c}\mathbf{5} \\
16.7 \%\end{array}$ & $\begin{array}{c}\mathbf{0} \\
0.0 \%\end{array}$ & $\begin{array}{c}\mathbf{0} \\
0.0 \%\end{array}$ & $\begin{array}{c}\mathbf{0} \\
0.0 \%\end{array}$ & $\begin{array}{c}\mathbf{0} \\
0.0 \%\end{array}$ & $\begin{array}{c}0 \\
0.0 \%\end{array}$ & $\begin{array}{l}100 \% \\
0.0 \%\end{array}$ \\
\hline 1.0 & $\begin{array}{c}\mathbf{0} \\
0.0 \%\end{array}$ & $\begin{array}{c}\mathbf{4} \\
13.3 \%\end{array}$ & $\begin{array}{c}\mathbf{0} \\
0.0 \%\end{array}$ & $\begin{array}{c}\mathbf{0} \\
0.0 \%\end{array}$ & $\begin{array}{c}\mathbf{0} \\
0.0 \%\end{array}$ & $\begin{array}{c}\mathbf{0} \\
0.0 \%\end{array}$ & $\begin{array}{l}100 \% \\
0.0 \%\end{array}$ \\
\hline 2.5 & $\begin{array}{c}\mathbf{0} \\
0.0 \%\end{array}$ & $\begin{array}{c}1 \\
3.3 \%\end{array}$ & $\begin{array}{c}\mathbf{5} \\
16.7 \%\end{array}$ & $\begin{array}{c}\mathbf{0} \\
0.0 \%\end{array}$ & $\begin{array}{c}\mathbf{0} \\
0.0 \%\end{array}$ & $\begin{array}{c}0 \\
0.0 \%\end{array}$ & $\begin{array}{l}83.3 \% \\
16.7 \%\end{array}$ \\
\hline 5.0 & $\begin{array}{c}\mathbf{0} \\
0.0 \%\end{array}$ & $\begin{array}{c}\mathbf{0} \\
0.0 \%\end{array}$ & $\begin{array}{c}\mathbf{0} \\
0.0 \%\end{array}$ & $\begin{array}{c}\mathbf{5} \\
16.7 \%\end{array}$ & $\begin{array}{c}0 \\
0.0 \%\end{array}$ & $\begin{array}{c}\mathbf{0} \\
0.0 \%\end{array}$ & $\begin{array}{l}100 \% \\
0.0 \%\end{array}$ \\
\hline 7.5 & $\begin{array}{c}\mathbf{0} \\
0.0 \%\end{array}$ & $\begin{array}{c}\mathbf{0} \\
0.0 \%\end{array}$ & $\begin{array}{c}\mathbf{0} \\
0.0 \%\end{array}$ & $\begin{array}{c}\mathbf{0} \\
0.0 \%\end{array}$ & $\begin{array}{c}\mathbf{5} \\
16.7 \%\end{array}$ & $\begin{array}{c}1 \\
3.3 \%\end{array}$ & $\begin{array}{l}83.3 \% \\
16.7 \%\end{array}$ \\
\hline 10.0 & $\begin{array}{c}\mathbf{0} \\
0.0 \%\end{array}$ & $\begin{array}{c}\mathbf{0} \\
0.0 \%\end{array}$ & $\begin{array}{c}\mathbf{0} \\
0.0 \%\end{array}$ & $\begin{array}{c}\mathbf{0} \\
0.0 \%\end{array}$ & $\begin{array}{c}\mathbf{0} \\
0.0 \%\end{array}$ & $\begin{array}{c}4 \\
13.3 \%\end{array}$ & $\begin{array}{l}100 \% \\
0.0 \%\end{array}$ \\
\hline & $\begin{array}{l}100 \% \\
0.0 \%\end{array}$ & $\begin{array}{l}80.0 \% \\
20.0 \%\end{array}$ & $\begin{array}{l}100 \% \\
0.0 \%\end{array}$ & $\begin{array}{l}100 \% \\
0.0 \%\end{array}$ & $\begin{array}{l}100 \% \\
0.0 \%\end{array}$ & $\begin{array}{l}80.0 \% \\
20.0 \%\end{array}$ & $\begin{array}{c}93.3 \% \\
6.7 \%\end{array}$ \\
\hline & 0 & 1.0 & $\begin{array}{c}2.5 \\
\text { Pred }\end{array}$ & $\begin{array}{r}5.0 \\
\text { cted I }\end{array}$ & $\begin{array}{c}7.5 \\
\text { abel }\end{array}$ & 10.0 & \\
\hline
\end{tabular}

Fig. 2: Confusion matrices results for the deltaE and CC methods are given in (a) and (b), respectively.

The overall performance of the two methods was computed by calculating detection accuracy, which is defined as the ratio of sum of diagonal elements of confusion matrix to the total number of data points. The detection accuracy of the CC method was obtained as $93.3 \%$ while it was $96.7 \%$ for the $\Delta \mathrm{E}^{*}$ method.

For further evaluation of the performance of the $\Delta \mathrm{E}^{*}$ and $\mathrm{CC}$ methods, metrics of classification report, referred to as precision, recall and f1-score were employed. Here, precision is the proportion of true positive events to the sum of true and false positive events in a classification task, recall is defined as the proportion of true positive events to the sum of true positive and false negative events, and f1-score is calculated using both the precision and the recall of the binary test to compute a total score, $\mathrm{f} 1$, which is equal to 1 for perfect precision and recall.
The precision, recall and f1-scores of the $\triangle \mathrm{E}^{*}$ and $\mathrm{CC}$ methods for automatically classifying MB are given in TABLE I, where f1-score is 0.97 and 0.94 , respectively.

The proposed approach offers promising applications for water monitoring based on smartphone. However, there are some drawbacks needs to be addressed in future works. It is assumed that training process considers only discrete levels of concentration, so that the unknown solution is also at a discrete level in test step. In the case of an unknown solution with an untrained concentration is tested, the result will be the closest discrete concentration level. Moreover, the experimental conditions such as smartphone camera setting or illumination conditions need to be same both in training and experimental steps. Otherwise, the colorimetric assays need to be re-trained.

TABLE I: CLASSIFICATION REPORT OF COLOR MATCHING METHODS

\section{DeltaE}

\begin{tabular}{|ccccc|}
$\begin{array}{c}\text { Assay } \\
\text { Concentration }\end{array}$ & Precision & Recall & f1-score & Support \\
$\mathbf{0}$ & 1 & 1 & 1 & 5 \\
\hline $\mathbf{1}$ & 0.8 & 1 & 0.9 & 5 \\
$\mathbf{2 . 5}$ & 1 & 0.83 & 0.92 & 5 \\
\hline $\mathbf{5}$ & 1 & 1 & 1 & 5 \\
\hline $\mathbf{7 . 5}$ & 1 & 1 & 1 & 5 \\
\hline $\mathbf{1 0}$ & 1 & 1 & 1 & 5 \\
\hline Avg/total & 0.97 & 0.97 & 0.97 & 30 \\
\hline \hline
\end{tabular}

\begin{tabular}{|ccccc|}
$\begin{array}{c}\text { Assay } \\
\text { Concentration }\end{array}$ & Precision & Recall & f1-score & Support \\
$\mathbf{0}$ & 1 & 1 & 1 & 5 \\
$\mathbf{1}$ & 0.8 & 1 & 0.9 & 5 \\
$\mathbf{2 . 5}$ & 1 & 0.83 & 0.92 & 5 \\
\hline $\mathbf{5}$ & 1 & 1 & 1 & 5 \\
$\mathbf{7 . 5}$ & 1 & 0.83 & 0.92 & 5 \\
\hline $\mathbf{1 0}$ & 0.8 & 1 & 0.9 & 5 \\
\hline Avg/total & 0.93 & 0.94 & 0.94 & 30 \\
\hline
\end{tabular}




\section{CONCLUSION}

In this paper, we have presented a smartphone platform that is able to monitor water quality in a portable, low-cost and user-friendly manner. Flash of the smartphone was used as a light source and spectrum view of the assays was captured by a smartphone after the light is passed through them. The pictures were taken in controlled environment with manual camera settings to make sure same conditions were maintained since our reference model was based on single reference image. After reference models were created and saved with their concentration values, the concentration level of unknown assays could be calculated with the use of proposed color matching algorithms. For quantitative evaluation of the proposed system, methylene blue assays were prepared in different concentration levels. The experimental results prove that the discrete concentration level of the individual assays is able to be reliably calculated. The limitations associated with the proposed platform such as calculation of discrete concentration level or capturing pictures in controlled environment could be an interesting direction for future work.

\section{REFERENCES}

[1] L. F. Capitán-Vallvey, N. Lopez-Ruiz, A. Martinez-Olmos, M. M. Erenas, and A. J. Palma, "Recent developments in computer vision-based analytical chemistry: A tutorial review," Analytica chimica acta, vol. 899, pp. 23-56, 2015. https://doi.org/10.1016/j.aca.2015.10.009

[2] E. K. Grasse, M. H. Torcasio, and A. W. Smith, "Teaching UV-Vis Spectroscopy with a 3D-Printable Smartphone Spectrophotometer," Journal of Chemical Education, vol. 93, pp. 146-151, 2015. https://doi.org/10.1021/acs.jchemed.5b00654

[3] F. Li, Y. Bao, D. Wang, W. Wang, and L. Niu, "Smartphones for sensing," Science Bulletin, vol. 61, pp. 190-201, February 012016. https://doi.org/10.1007/s11434-015-0954-1

[4] S. Schaefer, "Colorimetric water quality sensing with mobile smart phones," University of British Columbia, 2014.

[5] G. K. Ozdemir, A. Bayram, V. Klc, N. Horzum, and M. E. Solmaz, "Smartphone-based detection of dyes in water for environmental sustainability," Analytical Methods, vol. 9, pp. 579-585, 2017. https://doi.org/10.1039/C6AY03073D

[6] Y. Intaravanne and S. Sumriddetchkajorn, "Android-based rice leaf color analyzer for estimating the needed amount of nitrogen fertilizer," Computers and Electronics in Agriculture, vol. 116, pp. 228-233, 2015. https://doi.org/10.1016/j.compag.2015.07.005

[7] S. Dutta, D. Sarma, A. Patel, and P. Nath, "Dye-Assisted pH Sensing Using a Smartphone," IEEE Photonics Technology Letters, vol. 27, pp. 2363-2366. https://doi.org/10.1109/LPT.2015.2465132

[8] M. A. Hossain, J. Canning, S. Ast, K. Cook, P. J. Rutledge, and A. Jamalipour, "Combined "dual" absorption and fluorescence smartphone spectrometers," Optics letters, vol. 40, pp. 1737-1740, 2015. https://doi.org/10.1364/OL.40.001737

[9] Y. Wang, X. Liu, P. Chen, N. T. Tran, J. Zhang, W. S. Chia, et al., "Smartphone spectrometer for colorimetric biosensing," Analyst, vol. 141, pp. 3233-3238, 2016. https://doi.org/10.1039/C5AN02508G

[10] K. D. Long, H. Yu, and B. T. Cunningham, "Smartphone instrument for portable enzyme-linked immunosorbent assays," Biomedical optics express, vol. 5, pp. 3792-3806, 2014. https://doi.org/10.1364/BOE.5.003792

[11] M.-Y. Jia, Q.-S. Wu, H. Li, Y. Zhang, Y.-F. Guan, and L. Feng, "The calibration of cellphone camera-based colorimetric sensor array and its application in the determination of glucose in urine," Biosensors and Bioelectronics, vol. 74, pp. 1029-1037, 2015. https://doi.org/10.1016/j.bios.2015.07.072
[12] A. Y. Mutlu, V. Klc, G. K. Ozdemir, A. Bayram, N. Horzum, and M. E. Solmaz, "Smartphone-based colorimetric detection via machine learning," Analyst, vol. 142, pp. 2434-2441, 2017. https://doi.org/10.1039/C7AN00741H

[13] M. K. Morsy, K. Zór, N. Kostesha, T. S. Alstrøm, A. Heiskanen, H. El-Tanahi, et al., "Development and validation of a colorimetric sensor array for fish spoilage monitoring," Food Control, vol. 60, pp. 346-352, 2016. https://doi.org/10.1016/j.foodcont.2015.07.038

[14] N. Lopez-Ruiz, V. F. Curto, M. M. Erenas, F. Benito-Lopez, D. Diamond, A. J. Palma, et al., "Smartphone-based simultaneous $\mathrm{pH}$ and nitrite colorimetric determination for paper microfluidic devices," Analytical chemistry, vol. 86, pp. 9554-9562, 2014. https://doi.org/10.1021/ac5019205

[15] C. I. de I'Eclairage, "Recommendations on Uniform Color Spaces-Color Difference Equations, Psyhometric Color Terms. Supplement no. 2 to CIE Publication No. 15 (E-1.3. 1) 1971," TC, pp. 1-3, 1978.

[16] J. C.-C. Yuan, J. D. Brewer, E. A. Monaco, and E. L. Davis, "Defining a natural tooth color space based on a 3-dimensional shade system," The Journal of prosthetic dentistry, vol. 98, pp. 110-119, 2007. https://doi.org/10.1016/S0022-3913(07)60044-4

[17] Y. Lei, W. Luo, Y. Wang, and J. Huang, "Video sequence matching based on the invariance of color correlation," IEEE transactions on circuits and systems for video technology, vol. 22, pp. 1332-1343, 2012. https://doi.org/10.1109/TCSVT.2012.2201670 